Revista de Derecho Político, núm. 33, 1991, pp. 249-259

\title{
CRÓNICA PARLAMENTARIA DEL SENADO
}

\author{
POR \\ M. ${ }^{a}$ VICTORIA GARCIAA-ATANCE Y GARCIA DE MORA \\ Profesora Titular de Derecho Constitucional \\ UNED
}

«Perché possano continuare a conservare la loro influenza sulla mente umana, le antiche veritá devono essere riaffermate nelle lingua e secondo i concetti delle generazioni successive.»

Friedrich A. von HAYEK

La Societá Libera

Firenze 1969

El tratamiento de la presente Crónica del Senado versará sobre la segunda parte del primer período de sesiones de 1990, siendo además el segundo de la presente legislatura, que complementa, de esta manera, la Crónica del Senado presentada en nuestro anterior número, en el que por razones de distribución y encaje de la actividad desarrollada por la Cámara Alta, nos vimos precisados a proceder al desdoblamiento del primer período de sesiones de 1990.

Asi, estos dos números de nuestra Revista (32 y 33) reflejan de manera completa los trabajos efectuados por el Senado a lo largo de todo el primer período de sesiones de 1990. 


\section{PRÁCTICAS APLICATIVAS DE LA CONSTITUCIÓN}

\section{A. Prácticas aplicativas de la Constitución de carácter normativo}

En la presente fracción del primer periodo de Sesiones del Senado, no merece reseñarse práctica aplicativa alguna de la Constitución, por no haberse producido en la Cámara Alta ninguna que pueda ser incluida en esta categoría.

\section{B. Prácticas aplicativas de la Constitución de carácter no normativo}

\section{- Informe del Defensor del Pueblo.}

En sesión informativa la Comisión correspondiente a la gestión realizada por la institución del Defensor del Pueblo a lo largo de 1989. Diario de Sesiones, n. ${ }^{\circ}$ 17, Sesión en Comisión, de fecha 16 de mayo de 1990.

Este informe sería presentado partiendo de dos precisiones: en primer lugar un tratamiento metodológico de separar los derechos fundamentales y libertades públicas, así como las quejas sobre estas materias, de aquellas otras que se refieren al funcionamiento ordinario de las distintas Administraciones públicas, por la dispar importancia entre unas y otras.

En segundo lugar, el Defensor del Pueblo enfatizó en su propósito de que su Informe reflejara ante la Cámara no sólo las quejas de los ciudadanos, sino también la parte positiva sobre la situación de las Administraciones públicas.

Desde esta perspectiva se inició el Informe con la denuncia de algunas vulneraciones individuales de Derechos fundamentales, entre ellos se hizo referencia al articulo 15 de la CE: el derecho a la vida, a la integridad física y la interdicción de tratos degradantes. En este ámbito incluiría las novatadas del servicio militar, que producen daño a las personas no sólo en su dignidad, sino también físicamente.

Incluiria, asimismo, en este derecho fundamental del artículo 15, las quejas recibidas por los malos tratos inferidos por agentes de la autoridad, conducción y traslados de presos y detenidos, malos tratos en prisiones, etcétera.

Respecto a las quejas en torno al artículo $17 \mathrm{CE}$, han sido muy numerosas las recibidas en materia de privación de libertad, algunas de ellas derivadas de privaciones de libertad acordada judicialmente de manera 
anómala. En el ámbito de las Fuerzas de Seguridad también se han producido vulneraciones de detenciones sin aparente justificación, errores de identidad, etc.

En torno al derecho a la intimidad del artículo 18 de la CE, han sido frecuentes las quejas derivadas por el mal uso de las bases informáticas, el uso de las memorias informáticas.

Habiéndose detectado también muchas irregularidades al respecto, en torno al precepto constitucional de la inviolabilidad domiciliaria del artículo 18-4 CC, debido a la ambigüedad suscitada en relación a la consideración de determinados establecimientos públicos: hoteles, chabolas, etcétera, en concepto de domicilio.

Las infracciones en torno al artículo 24 han sido motivo frecuente de quejas, habiéndose vulnerado el derecho a la tutela judicial efectiva tanto por parte de la conducta individual de los jueces y titulares de los órganos, asi como por anomalias causadas por motivos estructurales o derivados de la propia normativa.

Otro de los aspectos fundamentales del Informe giraría en torno al procedimiento administrativo como garantía; en este sentido, los aspectos más reiterados por el Defensor del Pueblo serían: que se respete el principio de oficialidad en la actuación de la Administración; respecto al principio de publicidad, a través del acceso a archivos y registros.

Otro punto de especial relieve en orden a quejas residió en la mala calidad de servicios públicos: telefónica, correos, etc.

Por último, una especial atención al medio ambiente cuyo descuido requiere una mayor intervención de la policía administrativa, sancionadora en tales casos.

En términos generales, éste sería el testimonio del trabajo de la Institución del Defensor del Pueblo durante este ejercicio.

\section{ACTIVIDAD LEGISLATIVA}

En esta clasificación incluimos, como viene siendo costumbre, la actividad legislativa desarrollada tanto en Comisión como en Pleno, en lo referente a Proyectos de Ley así como Proposiciones de Ley. 


\section{A. Proyectos de Ley}

- Autorización del Pleno para la tramitación en lectura única del Proyecto de Ley de adaptación del concierto económico con la Comunidad Autónoma del Pais Vasco a la Ley Reguladora de Haciendas Locales y a la Ley de Tasas y Precios Públicos. Diario de Sesiones del Senado, n. ${ }^{\circ} 16$, Sesión Plenaria de fecha 8 de mayo de 1990.

- Lectura única del Proyecto de Ley remitido por el Congreso de los Diputados: Proyecto de Ley de adaptación del concierto económico con la Comunidad Autónoma del País Vasco a la Ley Reguladora de Haciendas Locales y a la Ley de Tasas y Precios Públicos. Diario de Sesiones del Senado, n. ${ }^{\circ}$ 18, Sesión en Comisión de fecha 22 de mayo de 1990.

- Proyecto de Ley sobre modificación del artículo $4 .^{\circ}$ de la Ley $50 /$ 1965, de 17 de julio, sobre venta de bienes a plazos. Diario de Sesiones del Senado, n. ${ }^{\circ}$ 21, Sesión Plenaria de fecha 6 de junio de 1990.

- Dictamen de la Comisión de Asuntos Exteriores en relación con el Convenio Marco Europeo sobre la Cooperación transfronteriza entre Entidades o Autoridades territoriales y del texto del Proyecto de declaración que España va a formular en el momento de la ratificación. Diario de Sesiones del Senado, n. ${ }^{\circ} 21$, de fecha 6 de junio 1990, Sesión Plenaria.

- Autorización del Pleno para la tramitación en lectura única del proyecto de Ley sobre negociación colectiva y participación en la determinación de las condiciones de trabajo de los empleados públicos. Diario de Sesiones del Senado, n. ${ }^{\circ} 23$, Sesión Plenaria de fecha 19 de junio de 1990.

- Dictamen de la Comisión de Presupuestos en relación con el Proyecto de Ley de Presupuestos Generales del Estado para 1990. Diario de Sesiones del Senado, . $^{\text {os }} 23,24$ y 25, Sesión Plenaria de fecha 19, 20 y 21 de junio 1990.

- Dictamen de la Comisión de Presupuestos en relación con el Proyecto de Ley de medidas en materia presupuestaria, financiera y tributaria. Diario de Sesiones del Senado, $n .^{\text {os }} 23,24$ y 25, Sesión Plenaria de fecha 19,20 y 21 junio 1990. 
- Lectura única del Proyecto de Ley sobre negociación colectiva. Diario de Sesiones del Senado, $n .^{\circ} 26$, en Sesión Plenaria de fecha 26 de junio 1990.

- Dictamen de la Comisión de Obras Públicas sobre el Proyecto de Ley de reforma del régimen urbanístico. Diario de Sesiones del Senado, n. ${ }^{\text {os }} 26$ y 27, en Sesión Plenaria de fecha 26 y 27 de junio 1990.

\section{B. Proposiciones de ley}

- Dictamen de la Comisión de Justicia en relación con la Proposición de Ley por la que se modifica la Ley 49/1960, de 21 de julio, de Propiedad Horizontal.

- Toma en consideración de la Proposición de. Ley Orgánica de Estatuto de Autonomía para Melilla. Diario de Sesiones del Senado, n. ${ }^{\circ} 19$, Sesión Plenaria de fecha 23 de mayo de 1990.

- Toma en consideración de Proposición de Ley Orgánica de Estatuto de Autonomía para Ceuta. Diario de Sesiones del Senado, n. ${ }^{\circ} 21$, Sesión Plenaria de fecha de 6 de junio 1990.

- Toma en consideración de la Proposición de Ley Orgánica relativa a la autorización y constitución de las Comunidades Autónomas de Ceuta y Melilla. Diario de Sesiones del Senado, $n .^{\circ} 28$, en Sesión Plenaria de fecha 28 de junio 1990.

\section{Tratados internacionales}

Conocimiento por el Pleno del Senado de Tratados y Convenios internacionales remitidos por el Pleno del Congreso de los Diputados.

- Conocimiento por el Pleno del Senado de la Enmienda al Convenio de 22 de noviembre de 1982, modificado y completado por protocolos de 10 de mayo. 
- Conocimiento por el Pleno de Tratados y Convenios internacionales remitidos por el Congreso de los Diputados, de los que nos vamos a limitar a dar, la referencia del Diario de Sesiones del Senado en que se recogen, para no hacer la relación excesivamente exhaustiva. Diario de Sesiones del Senado, n. ${ }^{\circ}$ 21, en Sesión Plenaria de fecha 6 de junio de 1990.

- Conocimiento por el Pleno de Tratados y Convenios remitidos por el Congreso: prórroga del Convenio Internacional del café, tramitándose por el procedimiento de urgencia, y canje de cartas, constitutivo de Acuerdo entre España y las Naciones Unidas, para la celebración del Simposio Internacional de integración de jovenes. Diario de Sesiones del Senado, número 26 , en Sesión Plenaria.

\section{ACTIVIDAD FINANCIERA}

- Dictamen y propuesta de resolución sobre la Cuenta General del Estado correspondiente a 1985, aprobados por la Comisión mixta para las relaciones con el Tribunal de Cuentas. Diario de Sesiones del Senado, n. ${ }^{\circ} 19$, Sesión Plenaria de fecha 23 de mayo de 1990.

- Dictamen sobre el Proyecto de Ley de Presupuestos Generales del Estado para 1990, sobre el Proyecto de Ley en materia de medidas financieras, presupuestarias y tributarias. Diario de Sesiones del Senado, n. ${ }^{\circ}$ 30, Sesión en Comisión de fecha 13 de junio de 1990.

- Dictamen de la Comisión de Presupuestos en relación con el Proyecto de Ley de Presupuestos Generales del Estado para 1990. Diario de Sesiones del Senado, n. ${ }^{\circ}$ 22, Sesión Plenaria de fecha 18 de junio de 1990.

- Dictamen de la Comisión de Presupuestos en relación con el Proyecto de Ley de medidas en materia presupuestaria, financiera y tributaria procedente del Real Decreto-Ley 7/1989, de 29 de diciembre. Diario de Sesiones del Senado, n. ${ }^{\text {ss }} 28$ y 29 , Sesión en Comisión de 11 y 12 de junio de 1990. 


\section{ACTIVIDAD DE CONTROL}

Al objeto de eludir una relación exhaustiva en torno a la actividad que ha desplegado el Senado en materia de control, procedemos, como viene siendo habitual, a agrupar esta actividad por categorias, limitándonos, en consecuencia, a indicar de forma referencial el Diario de Sesiones en que cada uno de estos medios de control quedan recogidos.

- Comparecencia del Secretario de Estado para la Cooperación Internacional y para Iberoamérica, para informar sobre la cooperación con Iberoamérica. Comisión de Asuntos Exteriores, Diario de Sesiones del Senado, n. ${ }^{\circ}$ 14, de fecha 3 de mayo de 1990.

- Diecisiete preguntas presentadas por los diferentes grupos parlamentarios repartidas del modo que sigue:

Siete preguntas del Grupo Socialista.

Seis preguntas del Grupo Popular.

Dos preguntas del Grupo Mixto.

Una pregunta del Grupo Catalán en el Senado de Convergencia y Unión.

Una pregunta del Grupo Centro Democrático y Social.

Diario de Sesiones del Senado, n. ${ }^{\circ} 16$, Sesión Plenaria de fecha 8 de mayo de 1990.

- Cuatro interpelaciones, de las que dos de ellas serían presentadas por el Grupo Popular y dos lo serían por el Grupo del Centro Democrático y Social. Diario de Sesiones del Senado n. ${ }^{\circ} 16$, Sesión Plenaria, fecha 8 de mayo 1990.

- Una interpelación por parte del Grupo mixto sobre adaptación de la estructura de los Ministerios y Organismos - dependientes de ellos-a las exigencias del proceso autonómico. Diario de Sesiones del Senado, n. ${ }^{\circ}$ 17, Sesión Plenaria de fecha 9 mayo de 1990. 
- Tres mociones: una de ellas presentada por el Grupo Mixto y dos por parte del Grupo Popular, instando al Gobierno a que se cumplan eficazmente los preceptos constitucionales en relación al uso, respeto y protección del castellano como lengua oficial, así como urgiendo al Gobierno a que se modifique y actualice la normativa vigente en materia de seguridad y accidentalidad laboral. Diario de Sesiones del Senado, n. ${ }^{\circ} 17$, Sesión Plenaria de fecha 9 de mayo de 1990.

- Comparecencia del Secretario de Estado de Educación para informar sobre la Formación Profesional. Diario de Sesiones del Senado, n. ${ }^{\circ}$ 15, Sesión en Comisión de fecha 10 mayo 1990.

- Comparecencia del Secretario de Promoción Industrial y Tecnologia para informar sobre planes para potenciar la competitividad de las empresas.-Diario de Sesiones del Senado, n. ${ }^{\circ} 16$, Sesión en Comisión, de fecha 10 mayo 1990.

- Veintiuna preguntas presentadas por los diferentes grupos parlamentarios, repartidas del modo siguiente:

Nueve por el Grupo Parlamentario Socialista.

Seis por parte del Grupo Popular.

Tres por el Grupo Catalán en el Senado dé Convergencia y Unión.

Dos por parte del Grupo Centro Democrático y Social.

Una por el Grupo Mixto.

Diario de Sesiones del Senado, n. ${ }^{\circ}$, Sesión del Pleno, fecha 22 de mayo 1990.

- Tres interpelaciones, de las que dos fueron presentadas por parte del Grupo Popular, sobre medidas para evitar perniciosos efectos medioambientales, así como sobre medidas del Gobierno en materia de política agroalimentaria; y una interpelación por el Grupo Catalán en el Senado de Convergencia y Unión, sobre la participación de las Comunidades Autónomas en el marco de la Comunidad Económica Europea. 
- Tres mociones al Gobierno presentadas dos de ellas por el Grupo Centro Democrático y Social por las que el Senado insta al Gobierno a que remita a las Cortes un Proyecto de Ley que regule la integración en la Escala Superior de los respectivos Cuerpos de oficiales de la Escala Especial que hayan obtenido su empleo en aplicación de lo dispuesto en los artículos 3,16 a) y 18 de la Ley $14 / 82$, de 5 de mayo, y por otra parte, instando al Gobierno a que remita a las Cortes un Proyecto de Ley añadiendo un apartado 3 al artículo 172 de la Ley 230/1963, de 28 de diciembre, Ley General Tributaria.

Y la tercera moción presentada por el Grupo Catalán en el Senado de Convergencia y Unión, sobre problemas que afectan a médicos españoles y comprometen la calidad asistencial sanitaria. Diario de Sesiones del Senado, n. ${ }^{\circ}$ 19, Sesión Plenaria de fecha 23 de mayo de 1990.

- Comparecencia del Secretario de Estado de Defensa y del Secretario de Estado de Administración Militar para contestar las preguntas pendientes de contestación oral planteadas por miembros de la Comisión. Diario de Sesiones del Senado, n. ${ }^{\circ}$ 18, Sesión en Comisión de fecha 24 de mayo de 1990.

- Comparecencia del Director general de Asuntos Religiosos para informar sobre los acuerdos de cooperación del Gobierno Español con confesiones religiosas. Diario de Sesiones del Senado, n. ${ }^{\circ} 19$, Sesión en Comisión de fecha 25 de mayo de 1990.

- Comparecencia de altos cargos de la Administración para informar a las preguntas de los Senadores previamente al debate del Proyecto de Ley de Presupuestos Generales del Estado para 1990. Diario de Sesiones del Senado, $n .^{\circ} 21$, Sesión en Comisión de fecha de 28 de mayo de 1990; Diario de Sesiones del Senado, n. ${ }^{\circ}$ 22, de 29 de mayo de 1990, Sesión en Comisión; Diario de Sesiones del Senado, n. $^{\circ} 23$, Sesión en Comisión de fecha 20 de mayo de 1990.

- Comparecencia del Ministro de Asuntos Exteriores para informar sobre la CSCE, y la respuesta del Gobierno sobre los cambios producidos en Europa. Diario de Sesiones del Senado, n. ${ }^{\circ} 24$, Sesión en Comisión de fecha 30 de mayo de 1990.

- Comparecencia de la Directora general del Instituto de la Mujer para informar sobre el Plan de la igualdad de la mujer. Diario de Sesiones del Senado, n. ${ }^{\circ} 25$, Sesión en Comisión de fecha 4 de junio de 1990. 
- Veintiuna preguntas presentadas por los distintos grupos parlamentarios. Diario de Sesiones del Senado, $n .^{\circ} 20$, Sesión Plenaria de fecha 5 de junio de 1990.

- Cinco interpelaciones, tres de ellas presentadas por el Grupo Popular, sobre criterios de la política del Gobierno en relación al sector agroalimentario; sobre política energética y sobre efectos perniciosos medioambientales. Diario de Sesiones del Senado, $n^{\circ}{ }^{\circ}$ 20, Sesión Plenaria de fecha 5 de junio de 1990; una interpelación del Grupo Catalán en el Senado de Convergencia y Unió sobre medidas del Gobierno en orden a homologación de medicamentos; y por último, una interpelación por parte del Grupo Mixto sobre medidas políticas para la conservación de la Naturaleza. Diario de Sesiones del Senado, ${ }^{\circ}{ }^{\circ}$ 20, de fecha 5 de junio de 1990.

- Comparecencia del Ministro de Sanidad y Consumo. Diario de Sesiones del Senado, n. ${ }^{\circ} 26$, de fecha 7 de junio de 1990 . Sesión en Comisión.

- Comparecencia del Ministro de Industria para informar sobre la situación de la Mediana y Pequeña Industria en el Mercado Común Europeo. Diario de Sesiones del Senado, n. ${ }^{\circ} 27$, Sesión en Comisión de fecha 7 de junio de 1990.

- Tres mociones, dos de ellas presentadas por el Grupo Popular, por las que se insta al Gobierno que remita a las Cortes un Proyecto de Ley de adaptación y modificación de las Leyes reguladoras de cada tributo, así como un Plan de reestructuración del sector ganadero. Y asimismo una moción presentada por el Grupo Mixto instando al Gobierno el cierre definitivo de la central nuclear de Vandellós. Diario de Sesiones del Senado, n. ${ }^{\circ}$ 21, Sesión Plenaria, de fecha 6 de junio de 1990.

- Comparecencia del Presidente de la Xunta de Galicia para informar acerca de cuestiones relativas a su Comunidad Autónoma, y del Secretario general de Turismo. Diario de Sesiones del Senado, n. ${ }^{\circ} 32$, Sesión en Comisión, de fecha 25 de junio de 1990.

- Diecisiete preguntas formuladas por los diferentes Grupos parlamentarios en el Senado. Diario de Sesiones del Senado, $n .{ }^{\circ} 26$, Sesión Plenaria de fecha 26 de junio de 1990. 
- Tres interpelaciones, de ellas dos presentadas por el Grupo parlamentario Popular, y la tercera por el Grupo de Centro Democrático y Social, para mejorar la situación de la Justicia en el orden contencioso-administrativo. Diario de Sesiones del Senado, n. ${ }^{\circ} 26$, Sesión Plenaria de fecha 26 de junio de 1990.

- Moción del Grupo del Centro Democrático y Social sobre ayudas a las explotaciones agrarias equiparables a las de la CEE. Diario de Sesiones del Senado, n. ${ }^{\circ}$ 28, Sesión Plenaria de fecha 28 de junio de 1990.

- Comparecencia del Director del Instituto para la Conservación de la Naturaleza; comparecencia del Presidente de la Comunidad Autónoma de La Rioja. Diario de Sesiones del Senado, n. ${ }^{\circ} 33$, Sesión en Comisión, de fecha 28 de junio de 1990.

- Comparecencia del Secretario general de Empleo. Diario de Sesiones del Senado, n. ${ }^{\circ} 35$, Sesión en Comisión. 